\title{
MIMO-Based Mode-Division Multiplexed Transmission over Multimode Fibers
}

\author{
Roland Ryf and Nicolas K. Fontaine \\ Bell Laboratories, Alcatel-Lucent, \\ 791 Holmdel-Keyport Rd, Holmdel, NJ, 07733, USA \\ Email: Roland.Ryf@Alcatel-Lucent.com
}

\begin{abstract}
We present results of combined mode- and wavelength multiplexed transmission over conventional graded-index multimode fibers. We selectively couple and receive up to 6 spatial modes of multimode-mode fibers with 3 and 6 and 45 spatial modes using photonic-lanterns based mode-couplers. The transmitted signals are recovered using MIMO digital signal processing.
\end{abstract}

\section{INTRODUCTION}

The transmission capacity of single-mode fibers (SMFs) has been growing for over three decades, and is now approaching the theoretical limits imposed by nonlinearities in combination with Shannon's capacity formula[1]. SpaceDivision multiplexing (SDM) over a single fiber is currently under renewed interest because of its potential to dramatically increase the capacity of a single fiber by more then an order of magnitude compared to SMFs. The capacity increase is achieved by transmitting independent data streams over individual spatial and polarization modes of a multimode-mode fiber (MMF), where the coupling between the fiber modes incurred during transmission is undone at the coherent receivers using multiple-input multiple-output (MIMO) digital signal processing (DSP) techniques. In this contribution we present recent long-distance transmission experiments performed in MMFs supporting 3 spatial modes (LP01, LP11a, and LP11b) and 6 spatial modes (LP01, LP11a, LP11b, LP21a, LP21b, and LP02), so called few-mode fibers (FMFs). The fibers, which are based graded index profiles, were optimized to minimize the modal differential group delay (DGD) between the modes, thus reducing the arrival time spread of the multiple transmitted signals. Additionally, the residual time spread is further reduced by combining multiple sections of FMFs with partially compensating DGD contributions. The transmission results were achieved using mode-couplers based on photonic lanterns[2], [3]. The photonic lanterns consist of an adiabatic transition between multiple single-mode waveguides into a single waveguide with modes that are closely matched to the modes of the FMF used for transmission. The concept is scalable to large number of spatial modes with theoretically negligible mode-dependent loss (MDL) and negligible overall insertion loss, and represents therefore an almost ideal mode coupler for MIMO-based SDM transmission over few-mode fibers.

In contrast to FMFs, multimode fibers supporting more than 50 spatial modes are traditionally paired with VCSELs and commonly used for the lowest cost short-reach interconnects over hundreds of meters. Laser-optimized 50- $\mu$ m OM4 MMF offers the benefit of favorable connector tolerances and provides standardized, effective modal bandwidth $>4.7 \mathrm{GHz}-$ $\mathrm{km}$ at $850 \mathrm{~nm}$. Stuart[4] proposed the use of spatial diversity to increase either capacity or transmission distance of multimode fibers and numerous schemes for detection, group multiplexing, and multiple-input multiple-output (MIMO) digital signal processing (DSP) have been proposed and demonstrated[5], [6], [7], [8], [9] nevertheless only with modest increase in transmission distance and capacity. The full capacity of the multimode fiber can be unleashed by selectively coupling into all modes of a subset of mode groups (for example the first 3 mode groups) of a MMF and subsequent MIMO DSP. Selective coupling is achieved using photonic-lantern mode multiplexers (PL-MUXs) just as for the few-mode fiber cased, followed by an additional mode filter that only transmits light for the desired sub set of groups. The principle was experimentally demonstrated in [10] by transmitting a 30 Gbaud QPSK signal over 6 spatial modes, two polarization, and 32 wavelength channels with a total line rate of $23 \mathrm{Tbit} / \mathrm{s}$. The principle is scalable to larger number of modes, opening the door for Pbit/s transmission over a single fiber core.

\section{GRAdED-INDEX MUltimode Fiber}

MMFs with graded refractive index profile (GI-MMFs) in particular, offer nominally small differential group delays (DGDs) (typically around 50 to $200 \mathrm{ps} / \mathrm{km}$ ) and can scale up to 45 or more spatial modes, while maintaining a standard cladding diameter of $125 \mu \mathrm{m}$. MMFs are compatible with conventional fusion splicers and existing cabling and connector technologies. The modes in GI-MMFs are in theory uncoupled, however fiber imperfections and bending cause coupling between modes. Also, the modes in a GI-MMF are separated in mode groups, where strong mode coupling is observed between modes within a group (which are degenerate modes in the weakly guiding approximation), and a relatively small mode coupling (typically around $8 \%$ for a $100 \mathrm{~km}$ long fiber) is observed between modes of different groups. The non-zero DGD, which can build up to considerable delays between spatial paths for long-distance transmission, can be mitigated using DGD compensation, where a MMF is followed by either a MMF or a device that has DGD values with inverted signs. The compensation is simple for FMFs supporting 3 spatial modes, where only one DGD value is present, and becomes more difficult when the number of modes is increased. DGDcompensated fiber transmission spans have been demonstrated for up to 6 spatial modes[11]. For larger number of modes new strategies are necessary. Since deployed fibers typically consists of multiple cables that are spliced every $\mathrm{km}$, it is conceivable to perform partial DGD compensation by selecting 

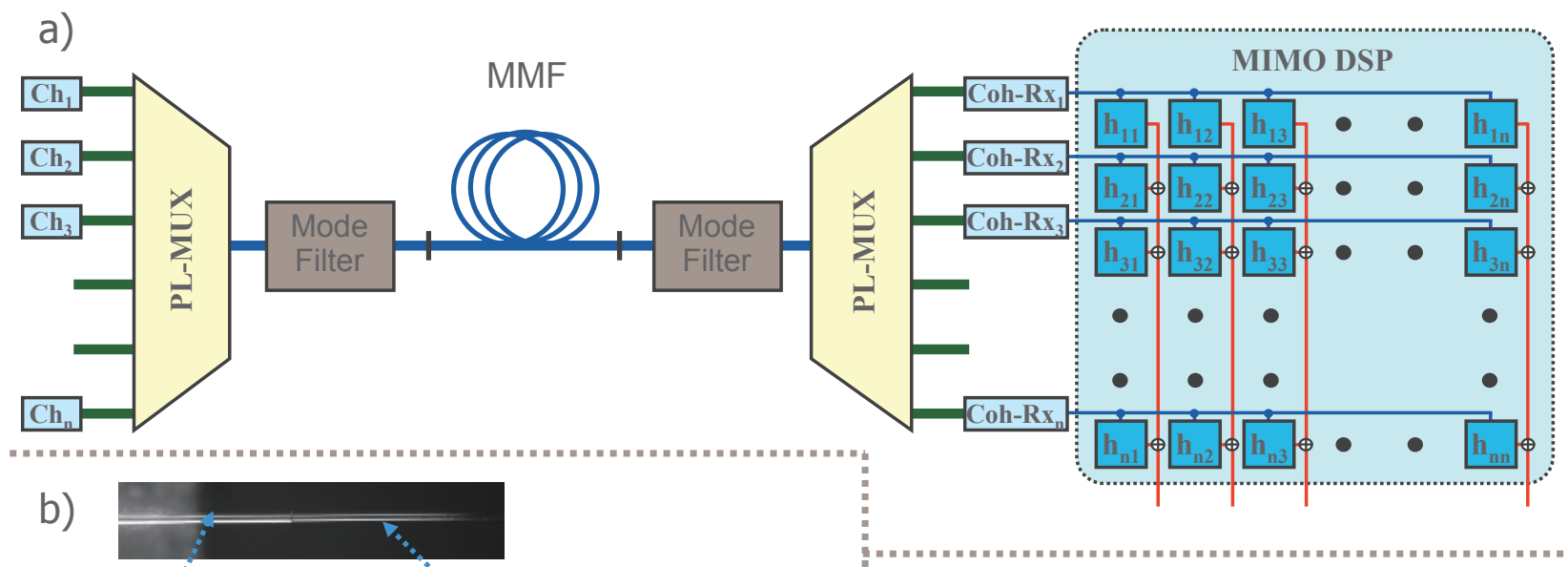

b)

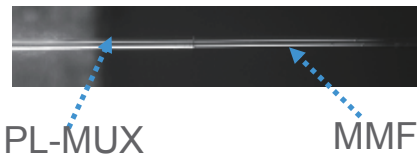

c)

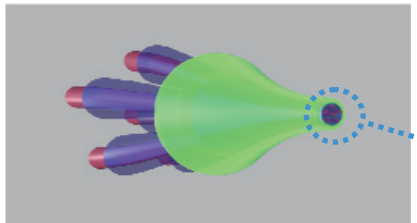

Loss 2-3dB, MDL 4-5dB

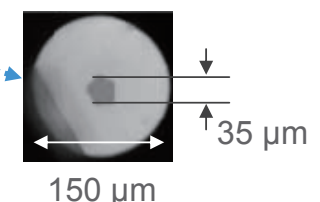

$150 \mu \mathrm{m}$

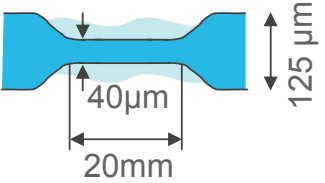

Fig. 1. a) Setup for a mode multiplexed single-span $N \times N$ MIMO transmission over MMF. b) Schematic design and cross section of a photonic-lantern mode coupler supporting 6 spatial modes. c) Mode filter to suppress unwanted modes used in MMF transmission.

the fiber segments according to their DGD properties and additionally intentionally introducing strong mode coupling at the splice. DGD compensated spans have been used in transmission experiments for distances up to $1500 \mathrm{~km}[12]$ and $700 \mathrm{~km}[11]$ for FMFs with 3 and 6 spatial modes, respectively.

\section{Mode-Division MUltipleXing OVER Multimode FIBERS}

A setup for single-span mode-division multiplexing transmission with $N$ spatial modes is shown in Fig. 1 a. The configuration uses two photonic-lantern multiplexers (PL-MUXs) to coupled and receive the modes of the MMF. A photograph of the photonic lantern side view and cross-section are shown in Fig. 1 b. The PL-MUXs are fabricated using a $\mathrm{CO}_{2}$ laser based glass processor, by adiabatically tapering single mode fibers enclosed in a low refractive index capillary from an initial diameter of $1.6 \mathrm{~mm}$ to $150 \mu \mathrm{m}$. The receiving PLMUX couples the received modes into $N$ single mode fibers (one for each detected spatial modes) and detected using $N$ polarization-diverse coherent receivers. The MIMO DSP, which functionally consists of an array of $N \times N$ linear equalizers[13], will the undo all linear impairments including the coupling between modes occurring in the MMF and the PL-MUXs.

When the PL-MUX can access all the modes supported by MMF no other optical element is necessary, more recently a scalable SDM transmission approach has been demonstrated[10], where a GI-MMF, supporting 45 modes, is utilized like a few-mode fiber, by using a double tapered fiber section as highly selective mode filter (see Fig. 1 c). The tapers were manufactured using a $\mathrm{CO}_{2}$ laser based glass processor. In the taper section, the fiber will only support the desired modes, whereas the higher order modes will leave the fiber through a refractive index matching coating placed on the cladding. The number of modes used for transmission can then be changed by replacing the mode filters while retaining the deployed fiber. The approach requires the coupling between the mode groups to stay moderate ( $<20 \%$ over the fiber span), and initial experiments confirm the validity of the approach for a $17 \mathrm{~km}$ long MMF[10].

\section{ACKNOWLEDGMENTS}

This work was partly supported by the IT R\&D program of MKE/KEIT. [10043383, Research of Mode-DivisionMultiplexing Optical Transmission Technology over $10 \mathrm{~km}$ Multi-Mode Fiber]

\section{REFERENCES}

[1] R.-J. Essiambre, et. al., J. Lightwave Technol., 28 (4), 662-701 (2010).

[2] N. K. Fontaine et. al. Opt. Express, 20 (24), 27123 (2012).

[3] S. G. Leon-Saval et. al.,Opt. Express, 18 (8), 8430 (2010).

[4] H.R. Stuart, Science, 289 (5477), 281-283 (2000).

[5] B. Franz and H. Bulow, PTL, 24 (16), 1363 (2012).

[6] A.R. Sha, et.al. J. Lightwave Technol., 23 (8), 2410 (2005).

[7] K. Shi; et.al., IEEE Summer topical 2013

[8] J. Carpenter, et. al., J. Lightwave Technol., 30 (24), 3946 (2012).

[9] C. Tsekrekos and A. Koonen, Opt. Lett. 32, 1041 (2007).

[10] R. Ryf et al. Proc. OFC 2014, PDP Th5B.1 (2014).

[11] R. Ryf et al. Proc. ECOC 2013, We.2.D.1 (2013).

[12] R. Ryf et al. Proc. OFC 2014, W4J.2 (2014).

[13] S. Randel et. al. Opt. Express, 19 (17), 16697 (2011). 